This is the author version of the following paper published by Nature on 27 May, 2015:

Ghahramani, Z. (2015) Probabilistic machine learning and artificial intelligence. Nature 521:452-459.

\title{
Probabilistic machine learning and artificial intelligence
}

\author{
Zoubin Ghahramani \\ University of Cambridge
}

May 28, 2015

\begin{abstract}
How can a machine learn from experience? Probabilistic modelling provides a framework for understanding what learning is, and has therefore emerged as one of the principal theoretical and practical approaches for designing machines that learn from data acquired through experience. The probabilistic framework, which describes how to represent and manipulate uncertainty about models and predictions, plays a central role in scientific data analysis, machine learning, robotics, cognitive science, and artificial intelligence. This article provides an introduction to this probabilistic framework, and reviews some state-of-the-art advances in the field, namely, probabilistic programming, Bayesian optimisation, data compression, and automatic model discovery.
\end{abstract}

\section{Introduction}

The key idea behind the probabilistic framework to machine learning is that learning can be thought of as inferring plausible models to explain observed data. A machine can use such models to make predictions about future data, and decisions that are rational given these predictions. Uncertainty plays a fundamental role in all of this. Observed data can be consistent with many models, and therefore which model is appropriate given the data is uncertain. Similarly, predictions, about future data and the future consequences of actions, are uncertain. Probability theory provides a framework for modelling uncertainty.

This article starts with an introduction to the probabilistic approach to machine learning and Bayesian inference, and then reviews some of the state-of-the-art in the field. The central thesis is that many aspects of learning and intelligence depend crucially on the careful probabilistic representation of uncertainty. Probabilistic approaches have only recently become a main-stream paradigm in artificial intelligence [1], robotics [2], and machine learning [3, 4]. Even now, there is controversy in these fields about how important it is to fully represent uncertainty. For example, 
recent advances using deep neural networks to solve challenging pattern recognition problems such as speech recognition [5], image classification [6,7], and prediction of words in text [8], do not overtly represent the uncertainty in the structure or parameters of those neural networks. However, my focus will not be on these types of pattern recognition problems, characterised by the availability of large amounts of data, but rather on problems where uncertainty is really a key ingredient, for example where a decision may depend on the amount of uncertainty.

In particular, I highlight five areas of current research at the frontiers of probabilistic machine learning, emphasising areas which are of broad relevance to scientists across many fields: (1) Probabilistic programming - a general framework for expressing probabilistic models as computer programs, which could have a major impact on scientific modelling; (2) Bayesian optimisation - an approach to globally optimising unknown functions; (3) Probabilistic data compression; (4) Automating the discovery of plausible and interpretable models from data; and (5) Hierarchical modelling for learning many related models, for example for personalised medicine or recommendation. While significant challenges remain, the coming decade promises substantial advances in artificial intelligence and machine learning based on the probabilistic framework.

\section{Probabilistic modelling and the representation of uncertainty}

At a most basic level, machine learning seeks to develop methods for computers to improve their performance at certain tasks based on observed data. Typical examples of such tasks might include detecting pedestrians in images taken from an autonomous vehicle, classifying gene-expression patterns from leukaemia patients into subtypes by clinical outcome, or translating English sentences into French. However, as we will see, the scope of machine learning tasks is even broader than these pattern classification or mapping tasks, and can include optimisation and decision making, compressing data, and automatically extracting interpretable models from data.

Data are the key ingredient of all machine learning systems. But data, even so called "Big Data", are useless on their own until one extracts knowledge or inferences from them. Almost all machine learning tasks can be formulated as making inferences about missing or latent data from the observed data - we will variously use the terms inference, prediction or forecasting to refer to this general task. Elaborating the example mentioned above, consider classifying leukaemia patients into one of the four main subtypes of this disease, based on each patient's measured gene-expression

patterns. Here the observed data are pairs of gene-expression patterns and labelled subtypes, and the unobserved or missing data to be inferred are the subtypes for new patients. In order to make inferences about unobserved data from the observed data, the learning system needs to make some assumptions; taken together these assumptions constitute a model. A model can be very simple 
and rigid, such as a classical statistical linear regression model, or complex and flexible, such as a large and deep neural network or even a model with infinitely many parameters. We return to this point in the next section. A model is considered to be well-defined if it can make forecasts or predictions about unobserved data having been trained on observed data (otherwise, if the model can't make predictions it can't be falsified, in the sense of Karl Popper, or as Wolfgang Pauli said the model is "not even wrong"). For example, in the classification setting, a well-defined model should be able to provide predictions of class labels for new patients. Since any sensible model will be uncertain when predicting unobserved data, uncertainty plays a fundamental role in modelling.

There are many forms of uncertainty in modelling. At the lowest level, model uncertainty is introduced from measurement noise, e.g., pixel noise or blur in images. At higher levels, a model may have many parameters, such as the coefficients of a linear regression, and there is uncertainty about which values of these parameters will be good at predicting new data. Finally, at the highest levels, there is often uncertainty about even the general structure of the model: is linear regression appropriate or a neural network, if the latter, how many layers, etc.

The probabilistic approach to modelling uses probability theory to express all forms of uncertainty [9]. Probability theory is the mathematical language for representing and manipulating uncertainty [10], in much the same way as calculus is the language for representing and manipulating rates of change. Fortunately, the probabilistic approach to modelling is conceptually very simple: probability distributions are used to represent all the uncertain unobserved quantities in a model (including structural, parametric, and noise-related) and how they relate to the data. Then the basic rules of probability theory are used to infer the unobserved quantities given the observed data. Learning from data occurs through the transformation of the prior probability distributions (defined before observing the data), into posterior distributions (after observing data). The application of probability theory to learning from data is called Bayesian learning (Box 1).

Apart from its conceptual simplicity, there are several appealing properties of the probabilistic framework for machine intelligence. Simple probability distributions over single or a few variables can be composed together to form the building blocks of larger more complex models. The dominant paradigm in machine learning over the last two decades for representing such compositional probabilistic models has been graphical models [11], with variants including directed graphs (a.k.a. Bayesian networks and belief networks), undirected graphs (a.k.a. Markov networks and random fields), and mixed graphs with both directed and undirected edges. A simple example of Bayesian inference in a directed graph is given in Figure 1. As we will see in a subsequent section, probabilistic programming offers an elegant way of generalising graphical models, allowing a much richer representations of models. The compositionality of probabilistic models means that the behaviour of these building blocks in the context of the larger model is often much easier to understand than, say, what will happen if one couples a nonlinear dynamical system (e.g., a recurrent neural network) 
to another. In particular, for a well-defined probabilistic model, it is always possible to generate data from the model; such "imaginary" data provide a window into the "mind" of the probabilistic model, helping us understand both the initial prior assumptions and what the model has learned at any later stage.

Probabilistic modelling also has some conceptual advantages over alternatives as a normative theory for learning in artificially intelligent (AI) systems. How should an AI system represent and update its beliefs about the world in light of data? The Cox axioms define some desiderata for representing beliefs; a consequence of these axioms is that "degrees of belief', ranging from "impossible" to "absolutely certain", must follow all the rules of probability theory [12, 10, 13]. This justifies the use of subjective Bayesian probabilistic representations in AI. An argument for Bayesian representations in AI that is motivated by decision theory is given by the Dutch-Book theorems. The argument rests on the idea that the strength of beliefs of an agent can be assessed by asking the agent whether it would be willing to accept bets at various odds (ratios of payoffs). The Dutch-Book theorems state that unless an AI system's (or human's, for that matter) degrees of beliefs are consistent with the rules of probability it will be willing to accept bets that are guaranteed to lose money [14]. Because of the force of these and many other arguments on the importance of a principled handling of uncertainty for intelligence, Bayesian probabilistic modelling has emerged not only as the theoretical foundation for rationality in AI systems but also as a model for normative behaviour in humans and animals $[15,16,17,18]$ (but see [19] and [20]), and much research is devoted to understanding how neural circuitry may be implementing Bayesian inference [21, 22].

Although conceptually simple, a fully probabilistic approach to machine learning poses a number of computational and modelling challenges. Computationally, the main challenge is that learning involves marginalising (i.e., summing out) all the variables in the model except for the variables of interest (c.f. Box 1). Such high-dimensional sums and integrals are generally computationally hard, in the sense that for many models there is no known polynomial-time algorithm for performing them exactly. Fortunately, a number of approximate integration algorithms have been developed, including Markov chain Monte Carlo (MCMC) methods, variational approximations, expectation propagation, and sequential Monte Carlo [23, 24, 25, 26]. It's worth noting that computational techniques are one area where Bayesian machine learning differs from much of the rest of machine learning: for Bayesians the main computational problem is integration, whereas for much of the rest of the community the focus is on optimisation of model parameters. However, this dichotomy is not as stark as it appears: many gradient-based optimisation methods can be turned into integration methods through the use of Langevin and Hamiltonian Monte Carlo methods [27, 28], while integration problems can be turned into optimisation problems through the use of variational approximations[24]. We revisit optimisation in a later section.

The main modelling challenge for probabilistic machine learning is that the model should be flexible 
enough to capture all the properties of the data required to achieve the prediction task of interest. One approach to addressing this challenge is to develop a prior that encompasses an open-ended universe of models that can adapt in complexity to the data. The key statistical concept underlying flexible models that grow in complexity with the data is nonparametrics.

\section{Flexibility through nonparametrics}

One of the lessons of modern machine learning is that the best predictive performance is often obtained from highly flexible learning systems, especially when learning from large data sets. Flexible models can make better predictions because to a greater extent they allow data to "speak for themselves". ${ }^{1}$ There are essentially two ways of achieving flexibility. The model could have a large number of parameters compared to the data set (for example, the neural network used recently to achieve near-state-of-the-art translation of English and French sentences is a probabilistic model with 384 million parameters [29]). Alternatively, the model can be defined using nonparametric components.

The best way to understand nonparametric models is through comparison to parametric ones. In a parametric model, there are a fixed finite number of parameters, and no matter how much training data are observed, all the data can do is set these finitely-many parameters that control future predictions. In contrast, nonparametric approaches have predictions that grow in complexity with the amount of training data, either by considering a nested sequence of parametric models with increasing numbers of parameters or by starting out with a model with infinitely many parameters. For example, in a classification problem, whereas a linear (i.e., parametric) classifier will always predict using a linear boundary between classes, a nonparametric classifier can learn a nonlinear boundary whose shape becomes more complex with more data. Many nonparametric models can be derived starting from a parametric model and considering that happens as the model grows to the limit of infinitely many parameters [30]. Clearly, fitting a model with infinitely many parameters to finite training data would result in "overfitting", in the sense that the model's predictions might reflect quirks of the training data rather than regularities that can be generalised to test data. Fortunately, Bayesian approaches are not prone to this kind of overfitting since they average over, rather than fit, the parameters (c.f. Box 1). Moreover, for many applications we have such huge data sets that the main concern is underfitting from the choice of an overly simplistic parametric model, rather than overfitting.

A review of Bayesian nonparametrics is outside the scope of this article (see for example [31, 32, 9]),

\footnotetext{
${ }^{1}$ But note that all predictions involve assumptions and therefore the data are never exclusively "speaking for themselves".
} 
but it's worth mentioning a few of the key models. Gaussian processes (GPs) are a very flexible nonparametric model for unknown functions, and are widely used for regression, classification, and many other applications that require inference on functions [33]. Consider learning a function relating the dose of some chemical to the response of an organism to that chemical. Instead of modelling this relationship with, say, a linear parametric function, a GP could be used to directly learn a nonparametric distribution of nonlinear functions consistent with the data. A notable example of a recent application of Gaussian processes is GaussianFace, a state-of-the-art approach to face recognition that outperforms humans and deep learning methods [34]. Dirichlet processes (DPs) are a nonparametric model with a long history in statistics [35] and are used for density estimation, clustering, time series analysis, and modelling the topics of documents [36]. To illustrate DPs, consider an application to modelling friendships in a social network, where each person can belong to one of many communities. A DP makes it possible to have a model where the number of inferred communities (i.e., clusters) grows with the number of people [37]. DPs have also been used for clustering gene expression patterns [38, 39]. The Indian buffet process (IBP) [40] is a nonparametric model which can be used for latent feature modelling, learning overlapping clusters, sparse matrix factorisation, or to nonparametrically learn the structure of a deep network [41]. Elaborating the social network modelling example, an IBP-based model allows each person to belong to some subset of a large number of potential communities (e.g., as defined by different families, workplaces, schools, hobbies, etc) rather than a single community, and the probability of friendship between two people depends on the number of overlapping communities they have [42]. In this case, the latent features of each person correspond to the communities, which are not assumed to be observed directly. The IBP can be thought of as a way of endowing Bayesian nonparametric models with "distributed representations" as popularised in the neural network literature [43]. An interesting link between Bayesian nonparametrics and neural networks is that, under fairly general conditions, a neural network with infinitely many hidden units is equivalent to a Gaussian process [44]. Note that the above nonparametric components should be thought of again as building blocks, which can be composed into more complex models as described in the introduction. The following section describes an even more powerful way of composing models, via probabilistic programming.

\section{Probabilistic Programming}

The basic idea in probabilistic programming it to use computer programs to represent probabilistic models $^{2}[45,46,47]$. One way to do this is for the computer program to define a generator for data from the probabilistic model, i.e., a simulator (Figure 2). This simulator makes calls to a random number generator in such a way that repeated runs from the simulator would sample

\footnotetext{
${ }^{2}$ http://probabilistic-programming.org
} 
different possible data sets from the model. This simulation framework is more general than the graphical model framework described previously since computer programs can allow constructs such as recursion (functions calling themselves) and control flow statements (e.g., if statements resulting in multiple paths a program can follow) which are difficult or impossible to represent in a finite graph. In fact, for many of the recent probabilistic programming languages that are based on extending Turing-complete languages (a class that includes almost all commonly-used languages), it is possible to represent any computable probability distribution as a probabilistic program [48].

The full potential of probabilistic programming comes from automating the process of inferring unobserved variables in the model conditioned on the observed data (c.f. Box 1). Conceptually, conditioning needs to compute input states of the program that generate data matching the observed data. Whereas normally we think of programs running from inputs to outputs, conditioning involves solving the inverse problem of inferring the inputs (in particular the random number calls) that match a certain program output. Such conditioning is performed by a universal inference engine, usually implemented by Monte Carlo sampling over possible executions over the simulator program that are consistent with the observed data. The fact that defining such universal inference algorithms for computer programs is even possible is somewhat surprising, but it is related to the generality of certain key ideas from sampling such as rejection sampling, sequential Monte Carlo [25], and "approximate Bayesian computation" [49].

As an example, imagine you write a probabilistic program that simulates a gene regulatory model relating unmeasured transcription factors to the expression levels of certain genes. Your uncertainty in each part of the model would be represented by the probability distributions used in the simulator. The universal inference engine can then condition the output of this program on the measured expression levels, and automatically infer the activity of the unmeasured transcription factors and other uncertain model parameters. Another application of probabilistic programming implements a computer vision system as the inverse of a computer graphics program [50].

There are several reasons why probabilistic programming could prove revolutionary for machine intelligence and scientific modelling. ${ }^{3}$ Firstly, the universal inference engine obviates the need to manually derive inference methods for models. Since deriving and implementing inference methods is generally the most rate-limiting and bug-prone step in modelling, often taking months, automating this step so that it takes minutes or seconds will massively accelerate the deployment of machine learning systems. Second, probabilistic programming could be potentially transformative for the sciences, since it allows for rapid prototyping and testing of different models of data. Probabilistic programming languages create a very clear separation between the model and the inference procedures, encouraging model-based thinking [51].

\footnotetext{
${ }^{3}$ Its potential has been noticed by DARPA which is currently funding a major programme called PPAML.
} 
There are a growing number of probabilistic programming languages. BUGS, STAN, AutoBayes, and Infer.NET $[52,53,54,55]$ allow only a restrictive class of models to be represented as compared to systems based on Turing-complete languages. In return for this restriction, inference in such languages can be much faster than for the more general languages, such as IBAL, BLOG, Figaro, Church, Venture, and Anglican [56, 57, 58, 59, 60, 61, 62]. A major emphasis of recent work is on fast inference in general languages (e.g., [63]). Nearly all approaches to probabilistic programming are Bayesian since it's hard to create other coherent frameworks for automated reasoning about uncertainty. Notable exceptions are systems like Theano, which is not itself a probabilistic programming language but uses symbolic differentiation to speed up and automate optimisation of parameters of neural networks and other probabilistic models [64].

While parameter optimisation is commonly used to improve probabilistic models, in the next section we will describe recent work on how probabilistic modelling can be used to improve optimisation!

\section{Bayesian optimisation}

Consider the very general problem of finding the global maximum of an unknown function which is expensive to evaluate (say, evaluating the function requires performing lots of computation, or conducting an experiment). Mathematically, for a function $f$ on a domain $\mathcal{X}$, the goal is to find a global maximiser $x^{*}$ :

$$
x^{*}=\arg \max _{x \in \mathcal{X}} f(x) .
$$

Bayesian optimisation poses this as a problem in sequential decision theory: where should one evaluate next so as to most quickly maximize $f$, taking into account the gain in information about the unknown function $f[65,66]$ ? For example, having evaluated at three points measuring the corresponding values of the function at those points, $\left\{\left(x_{1}, f\left(x_{1}\right)\right),\left(x_{2}, f\left(x_{2}\right)\right),\left(x_{3}, f\left(x_{3}\right)\right)\right\}$, which point $x$ should the algorithm evaluate next, and where does it believe the maximum to be? This is a classic machine intelligence problem with a wide range of applications in science and engineering, e.g., from drug design to robotics - where the function could be the drug's efficacy or the speed of a robot's gait respectively. Basically, it can be applied to any problem involving the optimisation of expensive functions; the qualifier "expensive" comes because Bayesian optimisation might use substantial computational resources to decide where to evaluate next, and these resources have to be traded off with the cost of function evaluations.

The current best-performing global optimisation methods maintain a Bayesian representation of the probability distribution over the uncertain function $f$ being optimised, and use this uncertainty to decide where (in $\mathcal{X}$ ) to query next $[67,68,69]$. In continuous spaces, most Bayesian optimisation methods use Gaussian processes (as described in the section on nonparametrics) to model 
the unknown function. An illustration of Bayesian optimisation is shown in Figure 3. A recent high-impact application has been in optimising the training process for machine learning models, including deep neural networks [70]. We can see this and related recent work [71] as further examples of the application of machine intelligence to improve machine intelligence

There are interesting links between Bayesian optimisation and reinforcement learning (RL). Specifically, Bayesian optimisation is a sequential decision problem where the decisions (choices of $x$ to evaluate) do not affect the state of the system (i.e., the actual function $f$ ). Such state-less sequential decision problems fall under the rubric of multi-arm bandits [72], a subclass of RL problems. More broadly, important recent work takes a Bayesian approach to learning to control uncertain systems [73] (and see the review [74]). Faithfully representing uncertainty about the future outcome of actions is particularly important in decision and control problems. Good decisions rely on good representations of the probability of different outcomes and their relative payoffs.

More generally, Bayesian optimisation is a special case of Bayesian numerical computation [75, 76] which is re-emerging as a very active area of research ${ }^{4}$, and also includes topics such as solving ordinary differential equations and numerical integration. In all these cases, probability theory is being used to represent computational uncertainty, that is, the uncertainty one has about the outcome of a deterministic computation.

\section{Data Compression}

Consider the problem of compressing data so as to communicate it or store it in as few bits are possible, in such a manner that the original data can be recovered exactly from the compressed data. Methods for such lossless data compression are ubiquitous in information technology, from computer hard drives to data transfer over the internet. Data compression and probabilistic modelling are two sides of the same coin, and Bayesian machine learning methods are increasingly advancing the state of the art in compression. The connection between compression and probabilistic modelling was established in Shannon's seminal work on the source coding theorem [77] which states that the number of bits required to losslessly compress data is bounded by the entropy of the probability distribution of the data. All commonly used lossless data compression algorithms (e.g., gzip, etc) can be viewed as probabilistic models of sequences of symbols.

The link to Bayesian machine learning is that the better the probabilistic model one learns, the higher the compression rate can be [78]. These models need to be flexible and adaptive, since different kinds of sequences have very different statistical structure (say, Shakespeare's plays, or computer source code). It turns out that some of the world's current best compression algorithms

\footnotetext{
${ }^{4}$ See http://www.probabilistic-numerics.org/
} 
(e.g., Sequence Memoizer, and PPM-DP) are equivalent to Bayesian nonparametric models of sequences, and improvements to compression are being made by understanding better how to learn the statistical structure of sequences $[79,80]$. Future advances in compression will come with advances in probabilistic machine learning, including special compression methods for non-sequence data such as images, graphs, and other structured objects.

\section{Automatically discovering interpretable models from data}

One of the grand challenges of machine learning is to fully automate the process of learning and explaining statistical models from data. This it the goal of the Automatic Statistician, a system that can automatically discover plausible models from data, and explain what it has discovered in plain English [81]. This could be useful to almost any field of endeavour that is reliant on extracting knowledge from data. In contrast to much of the machine learning literature which has been focused on extracting increasing performance improvements on pattern recognition problems using techniques such as kernel methods, random forests, or deep learning, the Automatic Statistician needs to build models that are composed of interpretable components, and to have a principled way of representing uncertainty about model structures given data. It also needs to be able to give reasonable answers not just for big data sets but also for small ones. Bayesian approaches provide an elegant way of trading off the complexity of the model and the complexity of the data, and probabilistic models are compositional and interpretable as described previously.

A prototype version of the Automatic Statistician takes in time-series data and automatically generates 5-15 page reports describing the model it has discovered (Figure 4$)^{5}$. This system is based on the idea that probabilistic building blocks can be composed together via a grammar to build an open-ended language of models [82]. In contrast to work on equation learning (e.g., [83]), the models attempt to capture general properties of functions (e.g., smoothness, periodicity, trends) rather than a precise equation. Handling uncertainty is at the core of the Automatic Statistician; it makes use of Bayesian nonparametrics to give it the flexibility to obtain state-of-the-art predictive performance, and the marginal likelihood (Box 1) is the key metric it uses to search the space of models.

Important earlier work includes statistical expert systems [84, 85] and the Robot Scientist, integrating machine learning and scientific discovery in a closed loop with an experimental platform in microbiology to automate the design and execution of new experiments [86]. AutoWeka is a recent project that automates learning classifiers, making heavy use of the Bayesian optimisation techniques described previously [71]. Efforts to automate the application of machine learning methods

\footnotetext{
${ }^{5}$ www.automaticstatistician.com
} 
to data have recently gained momentum, and may ultimately result in AI systems for data science.

\section{Perspectives}

The information revolution has resulted in the availability of ever larger collections of data. What is the role of uncertainty in modelling such "Big Data"? Classical statistical results state that under certain regularity conditions, in the limit of large data sets the posterior distribution of the parameters for Bayesian parametric models converges to a single point around the maximum likelihood estimate. Does this mean that Bayesian probabilistic modelling of uncertainty is unnecessary if you have a lot of data?

There are at least two reasons this is not the case [87]. First, as we have seen, Bayesian nonparametric models have essentially infinitely many parameters, so no matter how much data one has, their capacity to learn should not saturate, and their predictions should continue to improve.

A second reason is that many large data sets are in fact large collections of small data sets. For example, in areas like personalised medicine and recommendation systems, there might be a large amount of data, but there is still a relatively small amount of data for each patient or client, respectively. To customise predictions for each person it becomes necessary to build a model for each person - with its inherent uncertainties - and to couple these models together in a hierarchy so that information can be borrowed from other similar people. We call this the personalisation of models, and it is naturally implemented using hierarchical Bayesian approaches such as hierarchical Dirichlet processes [36], and Bayesian multi-task learning [88, 89].

Probabilistic approaches to machine learning and intelligence are a very active area of research with wide ranging impact beyond traditional pattern recognition problems. As we have outlined, these problems include data compression, optimisation, decision making, scientific model discovery and interpretation, and personalisation. The key distinction between problems in which a probabilistic approach is important and problems which can be solved using non-probabilistic machine learning approaches is whether uncertainty plays a central role. Moreover, most traditional optimisationbased machine learning approaches have probabilistic analogues which handle uncertainty in a more principled manner: for example, Bayesian neural networks represent the parameter uncertainty in neural networks [44], and mixture models are a probabilistic analogue for clustering methods [78]. While probabilistic machine learning often defines how to solve a problem in principle, the central challenge in the field is finding how in practice to do so in a computationally efficient manner [90, 91]. There are many approaches to the efficient approximation of computationally hard inference problems. Modern inference methods have made it possible to scale to millions of data points, making probabilistic methods computationally competitive with traditional methods 
[92, 93, 94, 95]. Ultimately, intelligence relies on understanding and acting in an imperfectly sensed and uncertain world. Probabilistic modelling will continue to play a central role in the development of ever more powerful machine learning and artificial intelligence systems.

\section{Acknowledgements}

ZG wishes to acknowledge EPSRC grant EP/I036575/1, the DARPA PPAML programme, a Google Focused Research Award for the Automatic Statistician, and generous support from Microsoft Research. Also acknowledged is valuable input from Hong Ge, Matthew W. Hoffman, Adam Ścibior, Max Welling, Daniel Wolpert, and the anonymous reviewers.

\section{References}

[1] Russell, S. \& Norvig, P. Artificial Intelligence: A Modern Approach (Prentice-Hall, Englewood Cliffs, NJ, 1995).

[2] Thrun, S., Burgard, W. \& Fox, D. Probabilistic Robotics (The MIT Press, 2006).

[3] Bishop, C. M. Pattern Recognition and Machine Learning (Springer, New York, 2006).

[4] Murphy, K. P. Machine learning: a probabilistic perspective (MIT press, 2012).

[5] Hinton, G. et al. Deep neural networks for acoustic modeling in speech recognition: The shared views of four research groups. Signal Processing Magazine, IEEE 29, 82-97 (2012).

[6] Krizhevsky, A., Sutskever, I. \& Hinton, G. E. Imagenet classification with deep convolutional neural networks. In Advances in neural information processing systems, 1097-1105 (2012).

[7] Sermanet, P. et al. Overfeat: Integrated recognition, localization and detection using convolutional networks. In International Conference on Learning Representations (ICLR 2014) (arXiv preprint arXiv:1312.6229, 2014).

[8] Bengio, Y., Ducharme, R., Vincent, P. \& Janvin, C. A neural probabilistic language model. The Journal of Machine Learning Research 3, 1137-1155 (2003).

[9] Ghahramani, Z. Bayesian nonparametrics and the probabilistic approach to modelling. Philosophical Transactions of the Royal Society A 371, 20110553 (2013).

[10] Jaynes, E. T. Probability theory: the logic of science (Cambridge University Press, 2003). 
There are two simple rules that underlie probability theory.

\begin{tabular}{|c|c|}
\hline $\begin{array}{l}\text { Sum rule: } \\
\text { Product rule: }\end{array}$ & $\begin{array}{c}P(x)=\sum_{y \in \mathcal{Y}} P(x, y) \\
P(x, y)=P(x) P(y \mid x)\end{array}$ \\
\hline
\end{tabular}

Here $x$ and $y$ correspond to observed or uncertain quantities, taking values in some sets $\mathcal{X}$ and $\mathcal{Y}$, respectively. For example, $x$ and $y$ might relate to the weather in Cambridge and London, respectively, both taking values in the set $\mathcal{X}=\mathcal{Y}=\{$ rainy, cloudy, sunny $\} . P(x)$ corresponds to the probability of $x$, which can be either a statement about the frequency of observing a particular value, or a subjective belief about it. $P(x, y)$ is the joint probability of observing $x$ and $y$, and $P(y \mid x)$ is the probability of $y$ conditioned on observing the value of $x$. The sum rule states that the marginal of $x$ is obtained by summing (or integrating for continuous variables) the joint over $y$. The product rule states that the joint can be decomposed as the product of the marginal and the conditional. Bayes rule is a corollary of these two rules:

$$
P(y \mid x)=\frac{P(x \mid y) P(y)}{P(x)}=\frac{P(x \mid y) P(y)}{\sum_{y \in \mathcal{Y}} P(x, y)} .
$$

We can apply probability theory to machine learning by replacing symbols above: We replace $x$ by $D$ to denote the observed data, we replace $y$ by $\theta$ to denote the unknown parameters of a model, and we condition all terms on $m$, the class of probabilistic models we are considering. We thus get:

\section{Learning:}

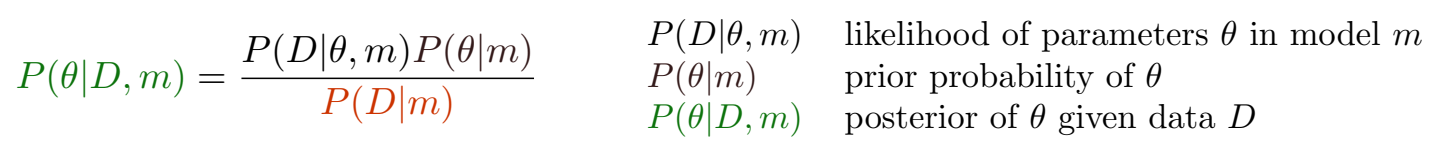

For example, the data $D$ might be a time-series of hourly observations of the weather in Cambridge and London, and the model might attempt to capture the joint weather patterns at both locations over successive hours, with parameters $\theta$ modelling correlations over time and space. Learning is the transformation of prior knowledge or assumptions about the parameters $P(\theta \mid m)$, via the data $D$, into posterior knowledge about the parameters, $P(\theta \mid D, m)$. This posterior is now the prior to be used for future data. A learned model can be used to predict or forecast new unseen test data, $D_{\text {test }}$, also by simply applying the sum and product rule:

\section{Prediction:}

$$
P\left(D_{\text {test }} \mid D, m\right)=\int P\left(D_{\text {test }} \mid \theta, D, m\right) P(\theta \mid D, m) d \theta
$$

Finally, different models can be compared by applying Bayes rule at the level of $m$ :

\section{Model Comparison:}

$$
\begin{aligned}
P(m \mid D) & =\frac{P(D \mid m) P(m)}{P(D)} \\
P(D \mid m) & =\int P(D \mid \theta, m) P(\theta \mid m) d \theta
\end{aligned}
$$

The term $P(D \mid m)$ is the marginal likelihood or model evidence, and implements a preference for simpler models known as Bayesian Ockham's Razor [96, 78, 97]. 


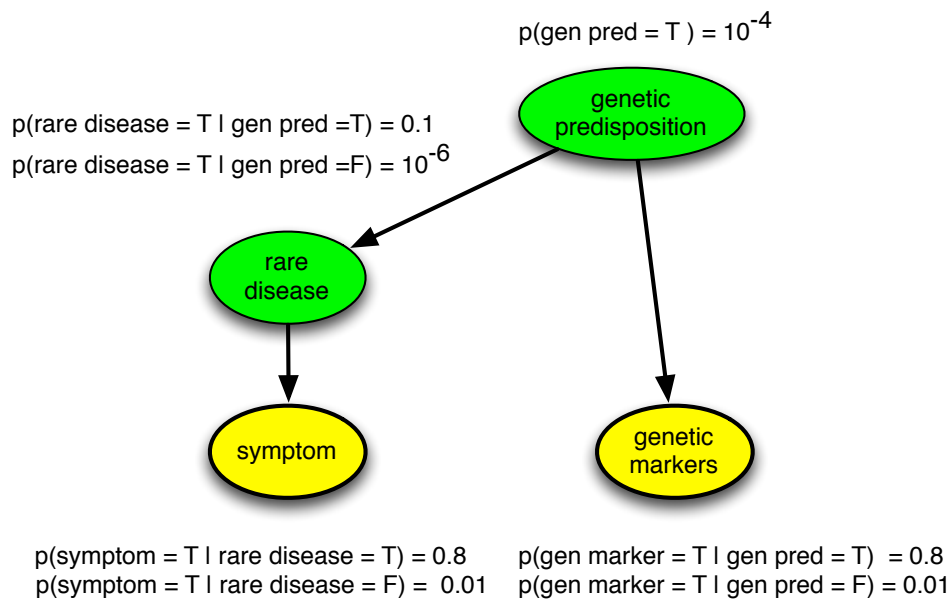

Figure 1: A simple example of Bayesian inference applied to a medical diagnosis problem. Here the problem is diagnosing a rare disease using information from the patient's symptoms and also potentially the patient's genetic marker measurements which indicate predisposition to this disease. In this example, all variables are assumed to be binary and $\mathrm{T}$ and $\mathrm{F}$ denote true and false, respectively. The relationships between variables are indicated by directed arrows and the probability of each variable given other variables they directly depend on is also shown. Yellow nodes denote measureable variables while green nodes denote hidden variables. Using the sum rule (Box 1), the prior probability of the patient having the rare disease is $p$ (rare disease $=T)=p($ rare disease $=$ $T \mid$ gen pred $=T) p($ gen $p r e d=T)+p($ rare disease $=T \mid$ gen pred $=F) p($ gen pred $=F)=1.1 \times 10^{-5}$. Applying Bayes rule we find that for a patient observed to have the symptom, the probability of the rare disease is $p($ rare disease $=T \mid$ symptom $=T)=8.8 \times 10^{-4}$, while for a patient observed to have the genetic marker it is $p($ rare disease $=T \mid$ gen marker $=T)=7.9 \times 10^{-4}$. Assuming that the patient has both the symptom and the genetic marker the probability of the rare disease increases to $p($ rare disease $=T \mid$ symptom $=T$, gen marker $=T)=0.06$. Here we have shown fixed, known model parameters, i.e., the numbers $\theta=\left(10^{-4}, 0.1,10^{-6}, 0.8,0.01,0.8,0.01\right)$. However, both these parameters and the structure of the model, i.e., the presence or absence of arrows and additional hidden variables, could be learned from a data set of patient records using the methods in Box 1 . 

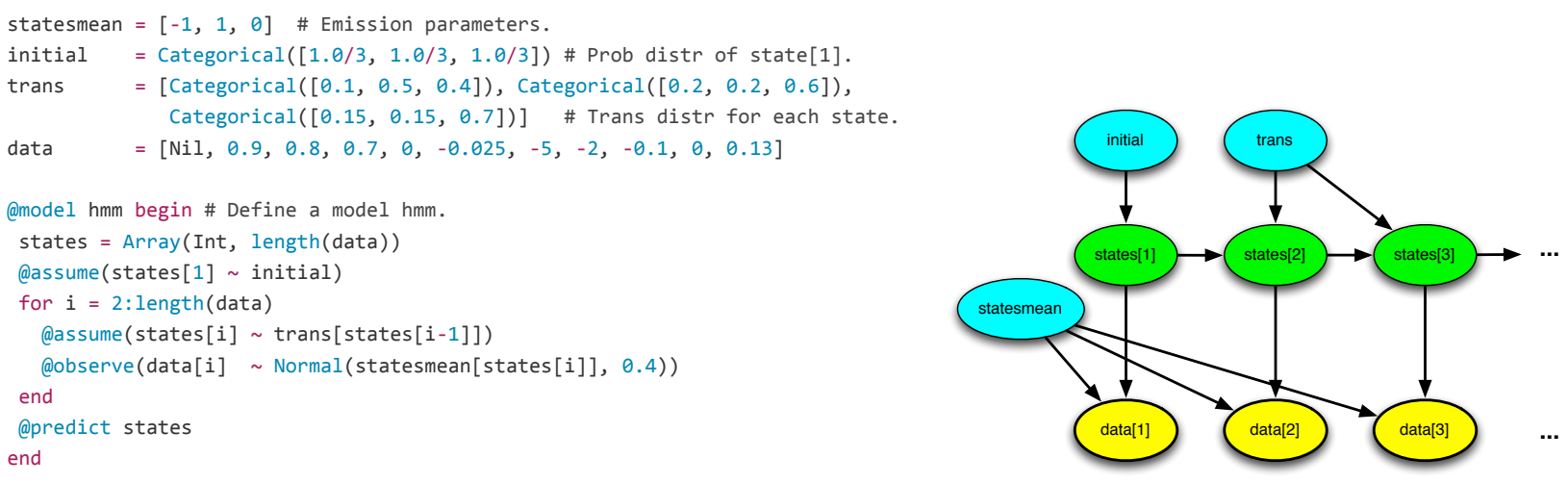

Figure 2: (a) A probabilistic program in Julia defining a simple 3-state hidden Markov model (HMM), inspired by an example in [62]. An HMM is a widely-used probabilistic model for sequential and time series data which assumes the data were obtained by transitioning stochastically between a discrete number of hidden states [98]. The first four lines define the model parameters and the data. Here trans is the $3 \times 3$ state-transition matrix, initial is the initial state distribution, and statesmean are the mean observations for each of the three states; actual observations are assumed to be noisy versions of this mean with Gaussian noise. The function hmm() starts the definition of the HMM, drawing the sequence of states with the Cassume statements, and conditioning on the observed data with the @observe statements. Finally @predict states that we wish to infer the states and data; this inference is done automatically via the universal inference engine which reasons over the configurations of this computer program. It would be trivial to modify this program so that the HMM parameters are unknown rather than fixed. (b) A graphical model corresponding to the HMM probabilistic program showing dependencies between the parameters (cyan), hidden state variables (green) and observed data (yellow). This graphical model highlights the compositional nature of probabilistic models. 

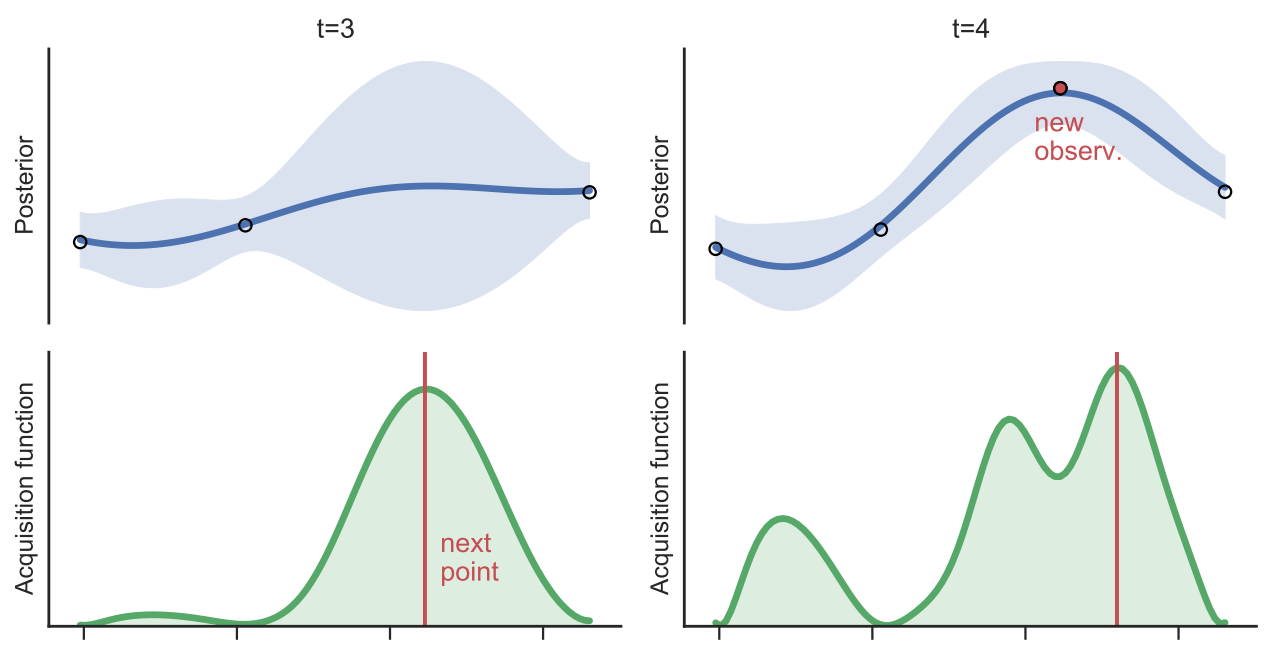

Figure 3: A simple illustration of Bayesian optimisation in one dimension. The goal is to maximise some true unknown function $f$ (not shown). Information about this function is gained by making observations (circles, top panels), which are evaluations of the function at specific $x$ values. These observations are used to infer a posterior distribution over the function values (shown as mean, blue line, and standard deviations, blue shaded area) representing the distribution of possible functions; note that uncertainty grows away from the observations. Based on this distribution over functions, an acquisition function is computed (green shaded area, bottom panels), which represents the gain from evaluating the unknown function $f$ at different $x$ values; note that the acquisition function is high where the posterior over $f$ has both high mean and large uncertainty. Different acquisition functions can be used such as "expected improvement" or "information-gain". The peak of the acquisition function (red line) is the best next point to evaluate, and is therefore chosen for evaluation (red dot, new observation). The left and right panels show an example of what could happen after three and four functions evaluations, respectively. 
(a)

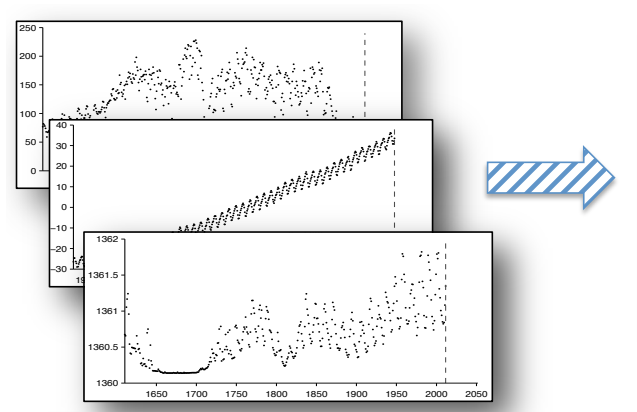

(b)

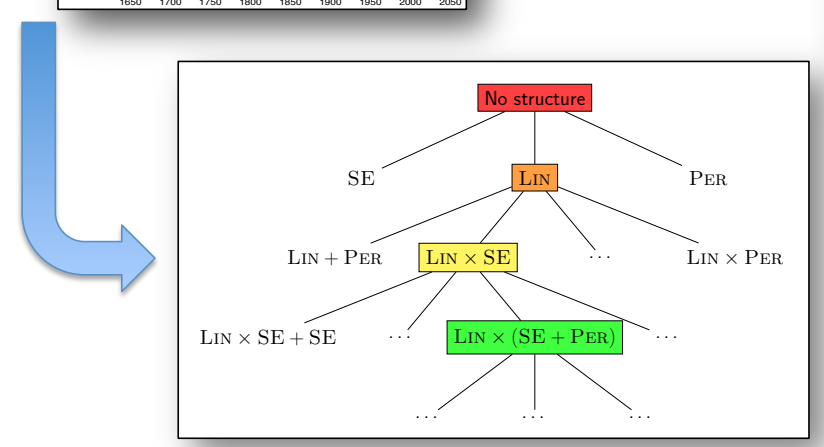

(d) This component is approximately periodic with a period of 10.8 years. Across periods the shape of
his function varies smoothly with a typical lengthscale of 369 years. The shape of this function hisis finction aries smoothly with a typical lenghthscale of 36.9 years. The shape of this function
within each period is very smooth and resembles a sinusoid. This component applies until 16433 and This component explains $71.5 \%$ of the residual variance; this increases the total variance explained from $72.8 \%$ to $92.3 \%$ from 0.18 to 0.15 .

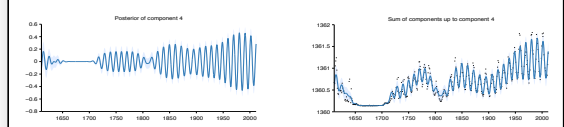

Figure 8: Pointwise posterior of component 4 (left) and the posterior of the cumulative sum or \begin{tabular}{|l|l|l|l|lll|}
\hline & Pigure 8: Pointwise postcrior of component 4 (left) and the posterior of the cumulative sum of \\
\hline & ACF & Periodogram & QQ & 150 & 200 & 250 \\
\hline
\end{tabular}

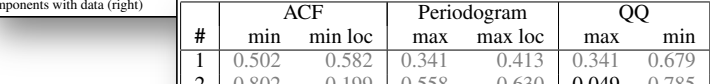
\begin{tabular}{l|llll|ll|l}
2 & 0.802 & 0.199 & 0.558 & 0.630 & 0.049 & 0.785 \\
\hline & 0.25 & 0.475 & 0.79 & 0.447 & 0.534 & 0.7696
\end{tabular} \begin{tabular}{lll|ll|ll||}
3 & 0.251 & 0.475 & 0.799 & 0.447 & 0.534 & 0.769 \\
4 & 0.527 & 0.503 & 0.504 & 0.481 & 0.430 & 0.686
\end{tabular} \begin{tabular}{l|ll|lll|l}
5 & 0.493 & 0.477 & 0.503 & 0.487 & 0.518 & 0.381 \\
\hline
\end{tabular}

\begin{tabular}{llll}
0.487 & 0.518 & 0.381 \\
\hline
\end{tabular}

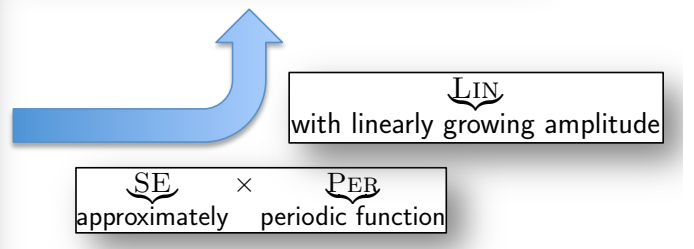

(c)

Figure 4: A flow diagram describing the Automatic Statistician. (a) The input to the system is data, in this case represented as time series. (b) The system searches over a grammar of models to discover a good interpretation of the data, using Bayesian inference to score models (Box 1). (c) Components of the model discovered are translated into English phrases. (d) The end result is a report with text, figures and tables, describing in detail what has been inferred about the data, including a section on model checking and criticism $[99,100]$. 
[11] Koller, D. \& Friedman, N. Probabilistic graphical models: Principles and techniques (The MIT Press, 2009).

[12] Cox, R. T. The algebra of probable inference (Johns Hopkins University Press, Baltimore, $\mathrm{MD}, 1961)$.

[13] Van Horn, K. S. Constructing a logic of plausible inference: a guide to Cox's theorem. International Journal of Approximate Reasoning 34, 3-24 (2003).

[14] De Finetti, B. La prévision: ses lois logiques, ses sources subjectives. In Annales de l'institut Henri Poincaré, vol. 7(1), 1-68 (Presses universitaires de France, 1937).

[15] Knill, D. \& Richards, W. Perception as Bayesian inference (Cambridge University Press, 1996).

[16] Griffiths, T. L. \& Tenenbaum, J. B. Optimal predictions in everyday cognition. Psychological Science 17, 767-773 (2006).

[17] Wolpert, D. M., Ghahramani, Z. \& Jordan, M. I. An internal model for sensorimotor integration. Science 269, 1880-1882 (1995).

[18] Tenenbaum, J. B., Kemp, C., Griffiths, T. L. \& Goodman, N. D. How to grow a mind: Statistics, structure, and abstraction. Science 331, 1279-1285 (2011).

[19] Marcus, G. F. \& Davis, E. How robust are probabilistic models of higher-level cognition? Psychological Science 24, 2351-2360 (2013).

[20] Goodman, N. D. et al. Relevant and robust a response to Marcus and Davis (2013). Psychological Science 0956797614559544 (2015).

[21] Doya, K., Ishii, S., Pouget, A. \& Rao, R. P. N. Bayesian Brain: Probabilistic Approaches to Neural Coding (The MIT Press, 2007).

[22] Deneve, S. Bayesian spiking neurons I: inference. Neural Computation 20, 91-117 (2008).

[23] Neal, R. M. Probabilistic inference using Markov chain Monte Carlo methods. Technical Report CRG-TR-93-1, Department of Computer Science, University of Toronto (1993).

[24] Jordan, M., Ghahramani, Z., Jaakkola, T. \& Saul, L. An introduction to variational methods in graphical models. Machine Learning 37, 183-233 (1999).

[25] Doucet, A., de Freitas, J. F. G. \& Gordon, N. J. Sequential Monte Carlo Methods in Practice (Springer-Verlag, New York, 2000). 
[26] Minka, T. P. Expectation propagation for approximate Bayesian inference. In Uncertainty in Artificial Intelligence, vol. 17, 362-369 (2001).

[27] Neal, R. M. MCMC using Hamiltonian dynamics. In S. Brooks A. Gelman, G. J. \& Meng, X.-L. (eds.) Handbook of Markov Chain Monte Carlo (Chapman \& Hall / CRC Press, 2010).

[28] Girolami, M. \& Calderhead, B. Riemann manifold Langevin and Hamiltonian Monte Carlo methods. Journal of the Royal Statistical Society: Series B (Statistical Methodology) 73, 123-214 (2011).

[29] Sutskever, I., Vinyals, O. \& Le, Q. V. Sequence to sequence learning with neural networks. In Ghahramani, Z., Welling, M., Cortes, C., Lawrence, N. \& Weinberger, K. (eds.) Advances in Neural Information Processing Systems 27, 3104-3112 (Curran Associates, Inc., 2014).

[30] Neal, R. M. Bayesian mixture modeling. In Maximum Entropy and Bayesian Methods, 197-211 (Springer Netherlands, 1992).

[31] Orbanz, P. \& Teh, Y. W. Bayesian nonparametric models. In Encyclopedia of Machine Learning (Springer, 2010).

[32] Hjort, N., Holmes, C., Müller, P. \& Walker, S. (eds.) Bayesian Nonparametrics (Cambridge University Press, 2010).

[33] Rasmussen, C. E. \& Williams, C. K. I. Gaussian Processes for Machine Learning (MIT Press, Cambridge, 2006).

[34] Lu, C. \& Tang, X. Surpassing human-level face verification performance on LFW with GaussianFace. In Proceedings of the 29th AAAI Conference on Artificial Intelligence (AAAI) (2015).

[35] Ferguson, T. S. A Bayesian analysis of some nonparametric problems. The Annals of Statistics 1, 209-230 (1973).

[36] Teh, Y. W., Jordan, M. I., Beal, M. J. \& Blei, D. M. Hierarchical Dirichlet processes. Journal of the American Statistical Association 101, 1566-1581 (2006).

[37] Kemp, C., Tenenbaum, J. B., Griffiths, T. L., Yamada, T. \& Ueda, N. Learning systems of concepts with an infinite relational model. In Proceedings of the 21st National Conference on Artificial Intelligence (2006).

[38] Medvedovic, M. \& Sivaganesan, S. Bayesian infinite mixture model based clustering of gene expression profiles. Bioinformatics 18, 1194-1206 (2002). 
[39] Rasmussen, C. E., De la Cruz, B. J., Ghahramani, Z. \& Wild, D. L. Modeling and visualizing uncertainty in gene expression clusters using Dirichlet process mixtures. Computational Biology and Bioinformatics, IEEE/ACM Transactions on 6, 615-628 (2009).

[40] Griffiths, T. L. \& Ghahramani, Z. The Indian buffet process: An introduction and review. Journal of Machine Learning Research 12, 1185-1224 (2011).

[41] Adams, R. P., Wallach, H. \& Ghahramani, Z. Learning the structure of deep sparse graphical models. In Teh, Y. W. \& Titterington, M. (eds.) 13th International Conference on Artificial Intelligence and Statistics, 1-8 (Chia Laguna, Sardinia, Italy, 2010). URL .

[42] Miller, K., Jordan, M. I. \& Griffiths, T. L. Nonparametric latent feature models for link prediction. In Advances in Neural Information Processing Systems, 1276-1284 (2009).

[43] Hinton, G. E., McClelland, J. L. \& Rumelhart, D. E. Distributed representations. In Parallel Distributed Processing: Explorations in the Microstructure of Cognition. Volume 1: Foundations, 77-109 (MIT Press, Cambridge, MA, 1986).

[44] Neal, R. M. Bayesian Learning for Neural Networks (Springer-Verlag, New York, 1996).

[45] Koller, D., McAllester, D. \& Pfeffer, A. Effective Bayesian inference for stochastic programs. In Proceedings of the 14th National Conference on Artificial Intelligence (AAAI) (1997).

[46] Goodman, N. D. \& Stuhlmüller, A. The Design and Implementation of Probabilistic Programming Languages (2015). Electronic, Retrieved 2015/3/22 from http://dippl.org.

[47] Pfeffer, A. Practical Probabilistic Programming (Manning Publications, 2015).

[48] Freer, C., Roy, D. \& Tenenbaum, J. B. Towards common-sense reasoning via conditional simulation: Legacies of Turing in artificial intelligence. In Turing's Legacy (ASL Lecture Notes in Logic) (2012).

[49] Marjoram, P., Molitor, J., Plagnol, V. \& Tavaré, S. Markov chain Monte Carlo without likelihoods. Proceedings of the National Academy of Sciences 100, 15324-15328 (2003).

[50] Mansinghka, V., Kulkarni, T. D., Perov, Y. N. \& Tenenbaum, J. Approximate Bayesian image interpretation using generative probabilistic graphics programs. In Advances in Neural Information Processing Systems 26, 1520-1528 (2013).

[51] Bishop, C. M. Model-based machine learning. Philosophical Transactions of the Royal Society A 371, 20120222 (2013).

[52] Lunn, D. J., Thomas, A., Best, N. \& Spiegelhalter, D. WinBUGS-a Bayesian modelling framework: concepts, structure, and extensibility. Statistics and Computing 10, 325-337 (2000). 
[53] Stan Development Team. Stan Modeling Language Users Guide and Reference Manual, Version 2.5.0 (2014). URL http://mc-stan.org/.

[54] Fischer, B. \& Schumann, J. AutoBayes: A system for generating data analysis programs from statistical models. Journal of Functional Programming 13, 483-508 (2003).

[55] Minka, T. P., Winn, J. M., Guiver, J. P. \& Knowles, D. A. Infer.NET 2.4 (2010). Microsoft Research Cambridge. http://research.microsoft.com/infernet.

[56] Pfeffer, A. IBAL: A probabilistic rational programming language. In IJCAI, 733-740 (2001).

[57] Milch, B. et al. BLOG: Probabilistic models with unknown objects. In Proc. 19th International Joint Conference on Artificial Intelligence (IJCAI), 1352-1359 (2005).

[58] Goodman, N., Mansinghka, V., Roy, D., Bonawitz, K. \& Tenenbaum, J. Church: a language for generative models. In Uncertainty in Artificial Intelligence, vol. 22, 23 (2008).

[59] Pfeffer, A. Figaro: An object-oriented probabilistic programming language. Tech. Rep., Charles River Analytics (2009).

[60] Wingate, D., Stuhlmüller, A. \& Goodman, N. D. Lightweight implementations of probabilistic programming languages via transformational compilation. In Artificial Intelligence and Statistics (2011).

[61] Mansinghka, V., Selsam, D. \& Perov, Y. Venture: a higher-order probabilistic programming platform with programmable inference. arXiv preprint (2014).

[62] Wood, F., van de Meent, J. W. \& Mansinghka, V. A new approach to probabilistic programming inference. In Proceedings of the 17th International conference on Artificial Intelligence and Statistics (2014).

[63] Li, L., Wu, Y. \& Russell, S. J. SWIFT: Compiled inference for probabilistic programs. Tech. Rep. UCB/EECS-2015-12, EECS Department, University of California, Berkeley (2015). URL http://www . eecs . berkeley .edu/Pubs/TechRpts/2015/EECS-2015-12.html.

[64] Bergstra, J. et al. Theano: a CPU and GPU math expression compiler. In Proceedings of the Python for Scientific Computing Conference (SciPy) (2010).

[65] Kushner, H. A new method of locating the maximum point of an arbitrary multipeak curve in the presence of noise. Journal of Basic Engineering 86, 97-106 (1964).

[66] Jones, D. R., Schonlau, M. \& Welch, W. J. Efficient global optimization of expensive blackbox functions. Journal of Global Optimization 13, 455-492 (1998). 
[67] Brochu, E., Cora, V. M. \& de Freitas, N. A tutorial on Bayesian optimization of expensive cost functions, with application to active user modeling and hierarchical reinforcement learning (2010). http://arXiv.org/abs/1012.2599.

[68] Hennig, P. \& Schuler, C. J. Entropy search for information-efficient global optimization. Journal of Machine Learning Research 13, 1809-1837 (2012).

[69] Hernández-Lobato, J. M., Hoffman, M. W. \& Ghahramani, Z. Predictive entropy search for efficient global optimization of black-box functions. In Advances in Neural Information Processing Systems (2014).

[70] Snoek, J., Larochelle, H. \& Adams, R. P. Practical Bayesian optimization of machine learning algorithms. In Advances in Neural Information Processing Systems (2012).

[71] Thornton, C., Hutter, F., Hoos, H. H. \& Leyton-Brown, K. Auto-WEKA: Combined selection and hyperparameter optimization of classification algorithms. In Proceedings of the 19th ACM SIGKDD International Conference on Knowledge Discovery and Data Mining, KDD '13, 847855 (ACM, New York, NY, USA, 2013).

[72] Robbins, H. Some aspects of the sequential design of experiments. Bulletin of the American Mathematical Society 55, 527-535 (1952).

[73] Deisenroth, M. P. \& Rasmussen, C. E. PILCO: A model-based and data-efficient approach to policy search. In 28th International Conference on Machine Learning (2011).

[74] Poupart, P. Bayesian reinforcement learning. In Encyclopedia of Machine Learning, 90-93 (Springer, 2010).

[75] Diaconis, P. Bayesian numerical analysis. In Statistical Decision Theory and Related Topics IV, vol. 1, 163-175 (Springer-Verlag, 1988).

[76] O'Hagan, A. Bayes-Hermite quadrature. Journal of Statistical Planning and Inference 29, 245-260 (1991).

[77] Shannon, C. \& Weaver, W. The Mathematical Theory of Communication (University of Illinois Press, Urbana, IL, 1949).

[78] MacKay, D. J. C. Information Theory, Inference, and Learning Algorithms (Cambridge University Press, 2003).

[79] Wood, F., Gasthaus, J., Archambeau, C., James, L. \& Teh, Y. W. The Sequence Memoizer. Communications of the ACM 54, 91-98 (2011). 
[80] Steinruecken, C., Ghahramani, Z. \& MacKay, D. J. C. Improving PPM with dynamic parameter updates. In Data Compression Conference (DCC 2015) (2015).

[81] Lloyd, J. R., Duvenaud, D., Grosse, R., Tenenbaum, J. B. \& Ghahramani, Z. Automatic construction and natural-language description of nonparametric regression models. In TwentyEighth AAAI Conference on Artificial Intelligence (AAAI-14) (2014).

[82] Grosse, R. B., Salakhutdinov, R. \& Tenenbaum, J. B. Exploiting compositionality to explore a large space of model structures. In Uncertainty in Artificial Intelligence (2012).

[83] Schmidt, M. \& Lipson, H. Distilling free-form natural laws from experimental data. Science 324, 81-85 (2009).

[84] Wolstenholme, D. E., O'Brien, C. M. \& Nelder, J. A. GLIMPSE: a knowledge-based front end for statistical analysis. Knowledge-Based Systems 1, 173-178 (1988).

[85] Hand, D. J. Patterns in statistical strategy. In Gale, W. A. (ed.) Artificial intelligence and statistics (1986).

[86] King, R. D. et al. Functional genomic hypothesis generation and experimentation by a robot scientist. Nature 427, 247-252 (2004).

[87] Welling, M. et al. Bayesian inference with Big Data: A snapshot from a workshop. In BIBiD (2014). URL http://www.ics.uci.edu/ welling/publications/papers/ISBA.pdf.

[88] Bakker, B. \& Heskes, T. Task clustering and gating for Bayesian multitask learning. Journal of Machine Learning Research 4, 83-99 (2003).

[89] Houlsby, N., Hernández-Lobato, J. M., Huszár, F. \& Ghahramani, Z. Collaborative Gaussian processes for preference learning. In Advances in Neural Information Processing Systems 26, 2096-2104 (Curran Associates, Inc., 2012).

[90] Russell, S. J. \& Wefald, E. Do the right thing: Studies in limited rationality (MIT press, 1991).

[91] Jordan, M. I. On statistics, computation and scalability. Bernoulli 19, 1378-1390 (2013).

[92] Hoffman, M., Blei, D., Paisley, J. \& Wang, C. Stochastic variational inference. Journal of Machine Learning Research 14, 1303-1347 (2013).

[93] Hensman, J., Fusi, N. \& Lawrence, N. D. Gaussian processes for big data. In Uncertainty in Artificial Intelligence (2013). 
[94] Korattikara, A., Chen, Y. \& Welling, M. Austerity in MCMC land: Cutting the MetropolisHastings budget. In Proceedings of the 31th International Conference on Machine Learning, ICML 2014, Beijing, China, 21-26 June 2014, 181-189 (2014).

[95] Paige, B., Wood, F., Doucet, A. \& Teh, Y. W. Asynchronous anytime sequential Monte Carlo. In Advances in Neural Information Processing Systems (2014).

[96] Jefferys, W. H. \& Berger, J. O. Ockham's Razor and Bayesian Analysis. American Scientist 80, 64-72 (1992).

[97] Rasmussen, C. E. \& Ghahramani, Z. Occam's Razor. In Advances in Neural Information Processing Systems 13, 294-300 (MIT Press, Cambridge, MA, 2001).

[98] Rabiner, L. R. A tutorial on hidden Markov models and selected applications in speech recognition. Proc. of the IEEE 77, 257-86. (1989).

[99] Gelman, A. et al. Bayesian Data Analysis, Third Edition (Chapman \& Hall/CRC Texts in Statistical Science. Taylor \& Francis, 2013).

[100] Lloyd, J. R. \& Ghahramani, Z. Statistical model criticism using kernel two sample tests (2015). http://mlg.eng.cam.ac.uk/Lloyd/papers/kernel-model-checking.pdf. 